CERN-TH/2000-319

$\mathrm{IFIC} / 00-63$

hep-ph/0010299

\title{
Minimalistic Neutrino Mass Model
}

\author{
André de Gouvêa \\ CERN - Theory Division \\ CH-1211 Genève 23, Switzerland \\ José W. F. Valle \\ Instituto de Física Corpuscular - C.S.I.C., Universitat de València, \\ Edificio Institutos de Paterna, Apt. 22085, E-46071 València, Spain
}

\begin{abstract}
We consider the simplest model which solves the solar and atmospheric neutrino puzzles, in the sense that it contains the smallest amount of beyond the Standard Model ingredients. The solar neutrino data is accounted for by Planck-mass effects while the atmospheric neutrino anomaly is due to the existence of a single righthanded neutrino at an intermediate mass scale between $10^{9} \mathrm{GeV}$ and $10^{14} \mathrm{GeV}$. Even though the neutrino mixing angles are not exactly predicted, they can be naturally large, which agrees well with the current experimental situation. Furthermore, the amount of lepton asymmetry produced in the early universe by the decay of the right-handed neutrino is very predictive and may be enough to explain the current baryon-to-photon ratio if the right-handed neutrinos are produced out of thermal equilibrium. One definitive test for the model is the search for anomalous seasonal effects at Borexino.
\end{abstract}




\section{Neutrino Puzzles and Masses}

The SuperKamiokande atmospheric neutrino data [1], provide very strong evidence for neutrino conversion, i.e. that neutrinos produced in well defined flavour eigenstates partially convert to different flavour eigenstates at the detection point. A similar conclusion follows from the long standing solar neutrino puzzle [2] and a number of other atmospheric neutrino experiments [3]. Altogether, solar and atmospheric data indicate the need for $\nu_{e}$ and $\nu_{\mu}$ conversions, respectively. There are a number of neutrino flavour conversion mechanisms 4 , 5, 6], and the simplest one is that of neutrino oscillations [7].

Although in some circumstances neutrino oscillations do not imply neutrino masses [8], typically they do. Due to the fact that neutrino mass and flavour eigenstates should, in general, differ, quantum interference effects can lead to sizeable neutrino conversion rates either from large mixing angles or from resonant matter effects. In this scenario, the solar and atmospheric neutrino puzzles are interpreted as strong evidence for nonzero neutrino masses.

The present data can be summarised as follows: the atmospheric neutrino puzzle is best solved by $\nu_{\mu} \rightarrow \nu_{\tau}$ with close to maximal mixing $\left(\sin ^{2} \theta \simeq 0.5\right)$ and $\Delta m_{\text {atm }}^{2} \simeq$ $10^{-3}-10^{-2}(\mathrm{eV})^{2}[9]$. On the other hand the solar neutrino puzzle requires $\nu_{e} \rightarrow \nu_{x}$ oscillations, where $\nu_{x}$ is some linear combination of $\nu_{\mu}$ and $\nu_{\tau}$. While the atmospheric neutrino data indicate large mixing angles, the current solar neutrino data do not indicate a unique solution. The mixing is either very small $\left(\sin ^{2} \omega \simeq 10^{-3}\right)$ or quite large $(0.1 \lesssim$ $\left.\sin ^{2} \omega \lesssim 0.5\right)$, while the value of $\Delta m_{\odot}^{2}$ varies from $10^{-4} \mathrm{eV}^{2}$ to $10^{-10} \mathrm{eV}^{2}$.

The neutrino oscillation interpretation of the solar and atmospheric data only fixes the mass-squared splittings amongst the the neutrinos, not the overall mass scale. In fact, the data are equally well explained if all neutrino masses were almost degenerate at some arbitrarily large value. There are, however, constraints on neutrino masses from tritium beta decay experiments at the level of $3 \mathrm{eV}$ [10], while cosmological considerations impose limits on the sum of all stable neutrino species, namely $\sum_{i} m_{\nu}^{i} \lesssim 30 \mathrm{eV}$. There are also a number of results from the non-observation of neutrinoless double beta decay [11], which imply that $M_{e e}=\sum_{i} U_{e i}^{2} m_{\nu_{i}} \lesssim 0.2 \mathrm{eV}$ where $\nu_{i}, i=1,2,3$ are the neutrino mass eigenstates, with masses $m_{\nu_{i}}$, and $U_{e i}$ are the components of $\nu_{e}$ in the $\nu_{i}$ basis $\left(\nu_{e}=\sum_{i} U_{e i} \nu_{i}\right)$.

Barring the possibility of quasi-degenerate neutrinos an/or exotic neutrino conversion 
mechanisms, all existing data fare easily satisfied if one assumes that the heaviest neutrino weighs around $0.05 \mathrm{eV}$ and is an almost fifty-fifty mixture of $\nu_{\mu}$ and $\nu_{\tau}$, while the second to heaviest neutrino weighs from $10^{-2} \mathrm{eV}$ to $10^{-5} \mathrm{eV}$ and is either composed of mostly the orthogonal combination of $\nu_{\mu}$ and $\nu_{\tau}$ with a very small $\nu_{e}$ component or an almost fifty-fifty mixture of this $\nu_{\mu}$ and $\nu_{\tau}$ linear combination and $\nu_{e}$. There is no preferred value for the mass of the lightest mass eigenstate, which can even be massless as far as the experimental constraints are concerned. In this case neutrino masses are very small, with the heaviest neutrino about $10^{7}$ times lighter than the lightest charged fermion.

What is wrong with neutrino masses? Naïvely, they are not allowed by the Standard Model (SM). This is very easy to see: given the field content and the gauge symmetries of the SM, it is impossible to write down a renormalizable neutrino mass term. However, if the SM is regarded as the effective low-energy limit of some, unknown, higher energy theory, neutrino Majorana masses are not forbidden and do arise after electroweak symmetry breaking. The neutrino Majorana mass comes from a dimension-5 term in the Lagrangian!

$$
\mathcal{L}_{5}=\frac{\lambda^{i j}}{M}\left(L^{i} H\right)\left(L^{j} H\right)
$$

such that, after electroweak symmetry breaking, one generates a neutrino mass matrix, $m_{\nu}^{i j}$ given by

$$
m_{\nu}^{i j}=\frac{\lambda^{i j} v^{2}}{M}
$$

Here $L^{i}$ is a lepton doublet field $(i, j=e, \mu, \tau), H$ is the Higgs doublet field and $M$ is an arbitrary mass scale. It is somehow related to the unknown high energy theory of which the SM is an effective theory. The $\lambda^{i j}$ are dimensionless couplings and $v=174 \mathrm{GeV}$ is the Higgs vacuum expectation value (vev). Note that Eq. (1.1) leads to a Majorana mass term which explicitly violates the lepton number symmetry, $L$. This is not a surprise since both lepton and baryon number $(B)$ are accidental global symmetries of the SM, and there is no reason they should be conserved by the higher energy theory. As a matter of fact, $B+L$ is violated in the $\mathrm{SM}$ via electroweak quantum effects, while $B-L$ is

${ }^{*}$ We will neglect throughout the LSND [12] result which, if confirmed by an independent experiment, would lead to the existence of light sterile neutrinos 13,14 . We therefore only consider the possibility of 3 light neutrino species.

${ }^{\dagger}$ An alternative approach generates Majorana neutrino masses by extending the SM Higgs sector to include exotic representations such as $S U(2)_{L}$ triplets with small vacuum expectation values [15]. We will not consider this approach here. 
still conserved. Furthermore, if string theory has anything to do with reality, it seems to predict that there are no exactly conserved global symmetries, and the only way to realize $B-L$ as a conserved symmetry is to assume it is gauged.

Alternatively, renormalizable neutrino Dirac masses are easily allowed within the SM if right-handed fermions uncharged under the SM gauge group exist. These are often referred to as right-handed neutrinos, and, even though they are not present in the SM, they can be added without causing any trouble (as they are SM singlets). Furthermore, their existence is quite natural in the context of a number of extended electroweak models as well as certain grand unified theories (GUTs) [16]. In their presence, the neutrinos acquire a Dirac mass like all the charged fermions, from a Yukawa coupling to the Higgs field

$$
\mathcal{L}_{L N H}=y^{i k} L^{i} N^{k} H+h . c .
$$

where $N^{k}(k=1 \ldots n$, where $n$ is the number of right-handed neutrinos) are right-handed fermions. As far as the SM gauge group is concerned, $n$ is completely unconstrained, since $N^{k}$ are $S U(2)_{L} \otimes U(1)$ singlets [15]. The $y^{i k}$ are the couplings of the sterile neutrinos to the lepton and Higgs doublet fields, leading to a neutrino Dirac mass matrix given by $y^{i k} v$.

A crucial fact is that right-handed neutrinos can also have Majorana masses, which are unprotected by the gauge symmetry. This means that the right-handed neutrino Majorana mass term comes from a renormalizable term in the Lagrangian (indeed from a relevant operator), which is completely unrelated to the electroweak symmetry breaking scale. The terms involving the right-handed neutrino are

$$
V_{N}=y^{i k} L^{i} N^{k} H+\frac{1}{2} M_{N}^{k l} N^{k} N^{l}+h . c .,
$$

where $M_{N}^{k l}$ is the right-handed neutrino Majorana mass matrix. The simultaneous presence of $y^{i k}$ and $M_{N}^{k l}$ in Eq. (1.4) implies that $L$ is violated.

In summary, the presence of right-handed neutrinos not only allows a Dirac mass term for the neutrino sector, but, in general, also violates $L$. Most importantly, it introduces a new energy scale into the SM Lagrangian. The task of neutrino mass modeling is then to explain the origin of Eq. (1.1) and of the $\lambda^{i j}$ and $M$ values or, alternatively, to determine the $y^{i k}$ and $M_{N}$ values in Eq. (1.4).

In this paper, we present a neutrino mass model which combines the above alternatives to generate neutrino masses and contains the least amount of new ingredients added to 
the SM. In Sec. 2 we define what is meant by "new ingredients" (or physics beyond the $\mathrm{SM}$ ), and argue in which sense the solar and atmospheric neutrino puzzles imply new physics. In Sec. 3 we present this "minimalistic" model and show how it satisfies the current experimental data. In Sec. 4 we discuss whether the minimalistic model allows for an explanation of the baryon-to-entropy ratio of the universe, via the elegant and economical mechanism of leptogenesis, and in Sec. 5 we conclude.

\section{2 "How Much" New Physics?}

Generating neutrino masses requires the addition of extra degrees of freedom and/or interactions to the SM [16], i.e., new physics. New physics is characterized by an energy scale above which the SM (and, perhaps, quantum field theory) is no longer appropriate and a different theory is required to describe physical processes involving this energy scale. This, of course, is what is meant by saying that the SM is the effective theory of some higher energy theory.

New physics usually manifests itself in the SM Lagrangian in the form of irrelevant operators, suppressed by powers of the scale of new physics. The presence of these operators can be detected either via the observation of rare or forbidden processes which are suppressed in the SM thanks to accidental symmetries which need not be preserved by the high energy theory, such as proton decay and $\mu \rightarrow e \gamma$, or via precision measurements of SM observables.

Independent of any hint for new physics, it is clear that the SM is an effective theory because it disregards the gravitational force, which certainly exists. This means that, if nothing else happens, the SM has to break down at the energy scale where gravitational effects become relevant, i.e., the Planck scale, $M_{P l} \simeq 10^{18} \mathrm{GeV}$. This means that, independent of whether there is some "intermediate" new physics, higher dimensional operators suppressed by the Planck scale should be present, unless forbidden by some deep, fundamental reason.

These " $1 / M_{P l}$ " operators are harmless. For example, a dimension-6 term $Q Q Q L / M_{P l}^{2}$ violates $B$ and $L$, and leads to proton decay. However, the expected proton lifetime due to this operator is many orders of magnitude away from the current experimental reach [17]. The same is true of operators which mediate $\mu \rightarrow e \gamma$ and other rare/forbidden processes.

As first noted by Barbieri, Ellis, and Gaillard [18] in the context of $S U(5)$ GUTS, and 
further explored by the authors of [19] in the case of the SM, "quantum gravity" effects also generate Eq. (1.1), namely,

$$
\mathcal{L}_{Q G}=\frac{\lambda^{i j}}{M_{P l}}\left(L^{i} H\right)\left(L^{j} H\right),
$$

should be considered as a "standard" term of the SM Lagrangian. What is most remarkable is that, for $\lambda^{i j}$ of order 1 , the entries of the Majorana neutrino mass matrix are of the order $\frac{(100)^{2}}{10^{18}} \times 10^{9} \mathrm{eV}=10^{-5} \mathrm{eV}$. This is in the acceptable mass range which solves the solar neutrino puzzle [9]! This implies the possibility that the solar neutrino puzzle can be solved without assuming any new physics between the electroweak and the Planck scale. In contrast, the atmospheric neutrino puzzle, when interpreted as evidence for a neutrino mass, is unambiguous proof of new physics, beyond the SM, at some intermediate energy scale.

An elegant way of understanding the smallness of neutrino masses is the seesaw mechanism [20], which takes advantage of Eq. (1.4). Since the right-handed neutrino mass scale is expected to be much larger than the electroweak scale it can be integrated out at energies smaller than this new mass scale, so that the effective Lagrangian again looks like Eq. (1.1), with

$$
m_{\nu}^{i j}=y^{i k}\left(M_{N}^{-1}\right)^{k l}\left(y^{T}\right)^{l j} \times v^{2} .
$$

The order of magnitude for the elements of $M_{N}$ required to solve the atmospheric neutrino puzzle is such that $M_{N}=O\left(\frac{(y v)^{2}}{10^{-10}} \mathrm{GeV}\right)$. For typical values of the Yukawa couplings $(y)$, one obtains $10^{9} \mathrm{GeV} \lesssim M_{N} \lesssim 10^{14} \mathrm{GeV}$.

There are many other, indirect, hints for new physics. Most importantly, there is the problem of the stability of the electroweak scale. Solving this so-called hierarchy problem, means, roughly, understanding why the electroweak scale (e.g. the SM Higgs mass) is so much smaller than the Planck scale. Its most popular solutions involve low-energy SUSY, which stabilizes scalar masses by eliminating quadratic divergences or, more recently, the existence of "large" extra dimensions [21]. The latter assumes that the fundamental scale of quantum gravity is indeed the weak scale, and that the apparent weakness of the gravitational force is due to the fact that gravity propagates in a multidimensional space-time, while SM fields are trapped in a four-dimensional space-time.

Other hints of new physics include the apparent unification of gauge couplings at around $10^{16} \mathrm{GeV}$, which seems to be an indication that the SM gauge group may be the "left-over" symmetry of some high energy GUT symmetry, and the hierarchy of the 
charged fermion masses which may be considered as a hint for horizontal flavour symmetries broken at intermediate energy scales.

In summary, there are many "theoretical" hints for new physics at very different energy scales, in particular the weak scale $\left(M_{W} \simeq 10^{2-3} \mathrm{GeV}\right)$ and the GUT scale $\left(M_{G U T} \simeq\right.$ $10^{16} \mathrm{GeV}$ ). All of these energy scales have been used to generate neutrino masses.

First note that one can generate Eq. (1.1) without invoking new mass scales above the weak scale, i.e. using $M=M_{W}$ in such a way that $\lambda^{i j}$ are very small. One example is SUSY with R-parity violation [22], where $\lambda^{i j}$ are given as a product of very suppressed couplings. Another possibility is to make use of the GUT scale and $1 / M_{P l}$ operators to induce right-handed neutrino masses of the order $M_{G U T}^{2} / M_{P l}$, such that the heaviest neutrino mass is of order $\frac{m_{t o p}^{2} M_{P l}}{M_{G U T}^{2}} \simeq 0.1 \mathrm{eV}[23]$.

In the case of SUSY, it has recently been pointed out that one can generate small Majorana or Dirac neutrino masses from SUSY breaking [24]. In these models, the neutrino masses are small for the same reason that the SUSY-preserving $\mu$-term is much smaller than the unknown high energy scale ( say $M_{P l}$ ).

Finally, theories with large extra dimensions can generate very small Dirac neutrino masses (Eq. (1.3) ) by assuming that the SM singlet fermions live in the extra dimensions and therefore their couplings in the SM "brane" are volume suppressed [25]. This means that neutrino masses are small for the same reason that the gravitational force is weak. Such theories, however, typically require that $L$ be conserved, unless it is broken in distant branes or by a totally different mechanism, such as R-parity violation [14. This is required in order to forbid terms similar to Eq. (2.1) but with $M=O(1) \mathrm{TeV}$, which would lead to unacceptably large neutrino masses.

It is clear that small neutrino masses are "expected" on theoretical grounds. However, the rich variety of theoretical options available illustrates that neither their scale nor the magnitude of neutrino mixing angles can be firmly predicted from first principles [16]. Our goal here is to focus on what appears to be the most economical way to solve the neutrino puzzles through neutrino-mass-induced oscillations, ignoring indirect beyond the SM hints. The motivation for this is two-fold: first, neutrino masses are the only direct experimental hint for beyond the SM physics and second, it is important to establish how contrived the new physics has to be in order to explain the current solar and atmospheric neutrino data. 


\section{The Minimalistic Model}

In order to minimize the amount of new physics invoked, we will take advantage of the already present quantum gravity contribution to neutrino masses, which naïvely implies that all three neutrinos weigh around $10^{-5} \mathrm{eV}$. Though not the favored solution, this is enough to account for the current solar neutrino data [9]. We need, however, to generate at least one neutrino mass of order $10^{-1} \mathrm{eV}$ in order to solve the atmospheric neutrino puzzle. The most economical way of doing this is to add one right-handed neutrino, at the appropriate mass scale $M_{N}$ [26]. . . This point was also emphasised in [28], where a single right-handed neutrino is used to explain the atmospheric data, while the solar data was explained by GUT scale effects.

Why one right-handed neutrino, and not three? First of all, in order to stick to the minimalistic approach, the smallest number of right-handed neutrinos is to be added. Second, and most important, there is, a priori, no correct number of right-handed neutrinos since, as gauge singlets, they do not contribute to SM gauge anomalies [15]. It is important to notice that these SM singlet right-handed fermions are very heavy and decouple from the low energy theory. Their only effect in the laboratory is indirect, through the generation of a Majorana neutrino mass, even though, as will be discussed in the next section, they may also play some role in the generation of the baryon number of the universe.

The SM Lagrangian with $1 / M_{P l}$ operators and one right-handed neutrino contains

$$
\frac{\lambda^{i j}}{M_{P l}}\left(L^{i} H\right)\left(L^{j} H\right)+y^{i} L^{i} N H+\frac{1}{2} M_{N} N N+h . c .
$$

Integrating out the right handed fermion, the neutrino mass matrix is (after electroweak symmetry breaking)

$$
m_{\nu}^{i j}=\left(\frac{\lambda^{i j}}{M_{P l}}+\frac{y^{i} y^{j}}{M_{N}}\right) v^{2} \equiv M_{1}+M_{3} .
$$

The parameters $\frac{y^{i} y^{j}}{M_{N}}$ and $\lambda^{i j}$, which are of order one, should be determined from the atmospheric and solar data. In order to simplify things, we rewrite $\frac{y^{i} y^{j}}{M_{N}} v^{2}$ as $m_{3} U_{i 3} U_{j 3}$, where $U_{e 3} \equiv \sin \xi, U_{\mu 3} \equiv \cos \xi \sin \theta$, and $U_{\tau 3} \equiv \cos \xi \cos \theta$, such that $\sum_{i}\left|U_{i 3}\right|^{2}=1$. The reason for this notation will become clear shortly.

* The fact that a single right-handed neutrino can solve the neutrino puzzles has been explored in the context of SUSY theories [27. Since these models also require supersymmetry, they are far from "minimalistic." 
Neglecting the $1 / M_{P l}$ term, the neutrino mass matrix is

$$
\mu_{\nu}=\left(\begin{array}{ccc}
U_{e 3}^{2} & U_{e 3} U_{\mu 3} & U_{e 3} U_{\tau 3} \\
U_{\mu 3} U_{e 3} & U_{\mu 3}^{2} & U_{\mu 3} U_{\tau 3} \\
U_{\tau 3} U_{e 3} & U_{\tau 3} U_{\mu 3} & U_{\tau 3}^{2}
\end{array}\right) m_{3}
$$

This matrix has a projective structure [29, 22], giving rise to one nonzero eigenvalue $m_{3}$ and two zero eigenvalues. However, since lepton number has been broken, there is no reason for any of the neutrino masses to be zero. Indeed, it is easy to see that nonzero masses to all neutrinos arise at the two-loop level. However, these are significantly smaller than the contribution from the $1 / M_{P l}$ operator we are considering here [30].

The eigenvector corresponding to the nonzero eigenvalue $m_{3}$ is $\vec{v}_{3} \equiv\left(U_{e 3}, U_{\mu 3}, U_{\tau 3}\right)^{\top}$ in the flavour basis which, because of unitarity, is determined by only two mixing angles [26], which we refer to as $\xi$ and $\theta$. This nonzero eigenvalue is the one that will be used to solve the atmospheric neutrino puzzle. If we choose $m_{3}=0.05 \mathrm{eV}, \sin \xi \simeq 0$ and $\cos ^{2} \theta \simeq \sin ^{2} \theta \simeq 0.5$, both the atmospheric neutrino data and the bounds from reactor neutrino experiments [31] are easily satisfied.

We now proceed to show that the addition of the $1 / M_{P l}$ term easily yields the just-so solution to the solar neutrino puzzle. For illustrative purposes, we initially assume that the $1 / M_{P l}$ term is "democratic," i.e.

$$
m_{\nu}=\left(\begin{array}{ccc}
U_{e 3}^{2} m_{3}+m & U_{e 3} U_{\mu 3} m_{3}+m & U_{e 3} U_{\tau 3} m_{3}+m \\
U_{\mu 3} U_{e 3} m_{3}+m & U_{\mu 3}^{2} m_{3}+m & U_{\mu 3} U_{\tau 3} m_{3}+m \\
U_{\tau 3} U_{e 3} m_{3}+m & U_{\tau 3} U_{\mu 3} m_{3}+m & U_{\tau 3}^{2} m_{3}+m
\end{array}\right),
$$

where $m=\frac{\lambda v^{2}}{M_{P l}}=\lambda \times 10^{-5} \mathrm{eV}$, and $\lambda$ is some order one coefficient. It is easy to see that Eq. (3.4) has one zero eigenvalue. This is due to the democratic nature of the $1 / M_{P l}$ operator. Note that one can rewrite Eq. (3.4) as

$$
m_{\nu}=\left(\begin{array}{cc}
U_{e 3} & \frac{1}{\sqrt{3}} \\
U_{\mu 3} & \frac{1}{\sqrt{3}} \\
U_{\tau 3} & \frac{1}{\sqrt{3}}
\end{array}\right)\left(\begin{array}{cc}
m_{3} & 0 \\
0 & 3 m
\end{array}\right)\left(\begin{array}{ccc}
U_{e 3} & U_{\mu 3} & U_{\tau 3} \\
\frac{1}{\sqrt{3}} & \frac{1}{\sqrt{3}} & \frac{1}{\sqrt{3}}
\end{array}\right),
$$

in such a way that it mimics the mass matrix one obtains in models with two righthanded neutrinos. Furthermore, in the case $m_{3}=0$, the mass matrix has only one massive eigenvalue, $m^{\prime} \equiv 3 m$ with corresponding eigenvector $\vec{v}_{1} \equiv(1 / \sqrt{3}, 1 / \sqrt{3}, 1 / \sqrt{3})^{\top}$.

The neutrino mass matrix given by Eq. 3.4 is simple to diagonalize since, as mentioned before, it has a zero eigenvalue and its corresponding eigenvector is trivial to compute. 
Note that $m_{\nu}=M_{3}+M_{1}$, such that $M_{3} \overrightarrow{v_{3}}=m_{3} \overrightarrow{v_{3}}$ and $M_{1} \overrightarrow{v_{1}}=m^{\prime} \overrightarrow{v_{1}}$. Furthermore, $M_{i} \vec{v}^{\perp}=0$, where $v^{\perp}$ is any vector orthogonal to $\vec{v}_{i}(i=1$ or 3$)$. Therefore, the massless eigenvector of $m_{\nu}$ is $\vec{V}_{0} \propto \vec{v}_{1} \times \vec{v}_{3}$. The remaining eigenvectors are going to be linear combinations of $\vec{v}_{1}$ and $\vec{v}_{3}$. It remains, therefore, to solve

$$
m_{\nu}\left(A \vec{v}_{1}+B \vec{v}_{3}\right)=\left(M_{1}+M_{3}\right)\left(A \vec{v}_{1}+B \vec{v}_{3}\right)=\Lambda\left(A \vec{v}_{1}+B \vec{v}_{3}\right)
$$

for $\Lambda, A$, and $B$ (of course, $A$ and $B$ are related by requiring the eigenvectors to be normalised). Defining $\vec{v}_{1} \cdot \vec{v}_{3} \equiv \cos \zeta$, the exact solution to Eq. (3.6) is

$$
\begin{aligned}
\left(\frac{A}{B}\right)^{ \pm} & =\frac{\Lambda^{ \pm}-m_{3}}{m_{3} \cos \zeta} \\
\Lambda^{ \pm} & =\frac{m^{\prime}+m_{3}}{2} \pm \frac{m^{\prime}+m_{3}}{2}\left[1-\frac{4 m^{\prime} m_{3}}{\left(m^{\prime}+m_{3}\right)^{2}} \sin ^{2} \zeta\right]^{\frac{1}{2}} .
\end{aligned}
$$

One can see that to first order in $\mathrm{m}^{\prime} / \mathrm{m}_{3}$ (the limit of interest here) the mass eigenvalues are, $\Lambda^{+}=m_{3}+m^{\prime} \cos ^{2} \zeta, \Lambda^{-}=m^{\prime} \sin ^{2} \zeta$, and $\Lambda^{0}=0$, with corresponding eigenvectors $\vec{V}^{+} \propto\left(\vec{v}_{3}+\frac{m^{\prime}}{m_{3}} \vec{v}_{1}\right), \vec{V}^{-} \propto\left(\vec{v}_{1}-\cos \zeta \vec{v}_{3}\right)$ and $\vec{V}^{0} \propto\left(\vec{v}_{1} \times \vec{v}_{3}\right)$.

Finally, we discuss what conditions must be met in order to solve the neutrino puzzles. First, the atmospheric data and the reactor constraints are, as expected, solved by choosing $\vec{v}_{3}$ and $m_{3}$ as in the previous case where the effect of the $1 / M_{P l}$ term was neglected $\left(m^{\prime}=0\right)$. The value of $\Delta m^{2}$ required for the "just-so" solution to the solar neutrino puzzle is also easily obtained for an order one $\lambda$ : note that $\sin ^{2} \zeta=1-\left(U_{e 3}+U_{\mu 3}+U_{\tau 3}\right)^{2} / 3$, which only vanishes if all $U_{i 3}=1 / \sqrt{3}$, which is not allowed by the atmospheric data [9]. In the extreme case of $\sin ^{2} \xi=0.1$ and $\sin ^{2} \theta=0.5$, one has $\sin ^{2} \zeta \simeq 0.1$, and $\Lambda^{-} \simeq 0.3 \lambda \times 10^{-5} \mathrm{eV}$, which is $O\left(10^{-5}\right)$ for an $O(1)$ value of $\lambda$.

A more serious concern is to guarantee large mixing in the solar sector. This can be read of from $U_{e 1} \equiv \cos \xi \cos \omega$, where $\omega$ is the "solar" mixing angle. Ordering the mass eigenstates in the order of increasing mass, $U_{e 1}=\left(\vec{V}_{0}\right)_{1}=\left(U_{\mu 3}-U_{\tau 3}\right) /(\sqrt{3} \sin \zeta)$. This vanishes if $U_{\mu 3}=U_{\tau 3}$, which implies $\cos \omega=0$, which is unacceptable. The situation is easily remedied, however, by requiring $U_{\mu 3}$ and $U_{\tau 3}$ to have opposite signs.

Though there are (loose) arguments in favour of a democratic $1 / M_{P l}$ contribution to the neutrino Majorana mass matrix, there is no deep reason for it. The agreement between the neutrino puzzles and the model proposed here does not depend on democratic dimension-5 operators, but is a rather generic feature of random $1 / M_{P l}$ coefficients. In order to check whether this is really the case, we performed a numerical diagonalization 


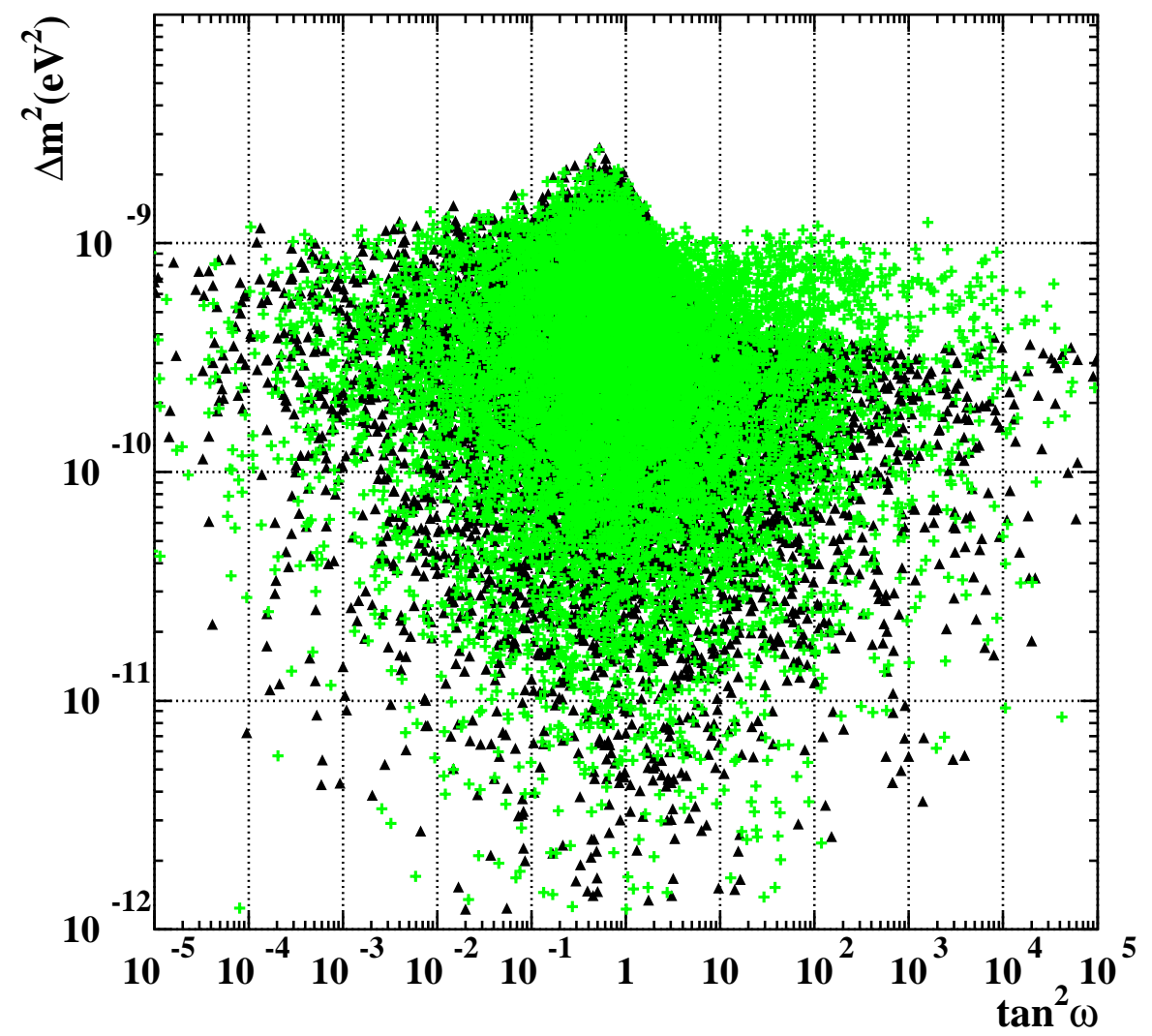

Figure 1: 10000 values of $\Delta m_{\odot}^{2}$ and $\tan ^{2} \omega$ obtained by randomly generated elements for the $M_{1}$ matrix (see text), for $\sin ^{2} \theta=0.5, \sin ^{2} \xi=0$ (dark triangles) or $\sin ^{2} \theta=0.5$, $\sin ^{2} \xi=0.1$ (light crosses), and $m_{3}=0.05 \mathrm{eV}$.

of Eq. (3.2) with random $\lambda^{i j}$ matrices and choosing $y^{i}$ such that the the atmospheric neutrino data is satisfied. Fig. 1 depicts the values obtained for $\Delta m_{\odot}^{2}$ and $\tan ^{2} \omega$ (where $\omega$ is the "solar" angle) for 10000 randomly generated $\lambda^{i j}$ (with elements between -2 and 2), for $\sin ^{2} \theta=0.5, m_{3}=0.05 \mathrm{eV}$ and $\sin ^{2} \xi=0$ (dark triangles) or $\sin ^{2} \xi=0.1$ (light crosses). It should be noted that close to maximal mixing $\left(\tan ^{2} \omega \sim 1\right)$ is preferred, and that the values of $\Delta m_{\odot}^{2}$ obtained agree quite well with the small $\Delta m_{\odot}^{2}$ range obtained in [9]. The situation remains the same if extra complex phases are included at random.

One possible criticism to the minimalistic model is the fact that, even though the solar data is naturally satisfied for random " $1 / M_{P l}$ " operators, we are required to not only choose the mass scale for the right-handed neutrino but also the relative values of the Yukawa couplings in order to satisfy the atmospheric neutrino data. This is not correct, and we proceed to argue that randomly generated $U_{i 3}$ easily satisfy not only 

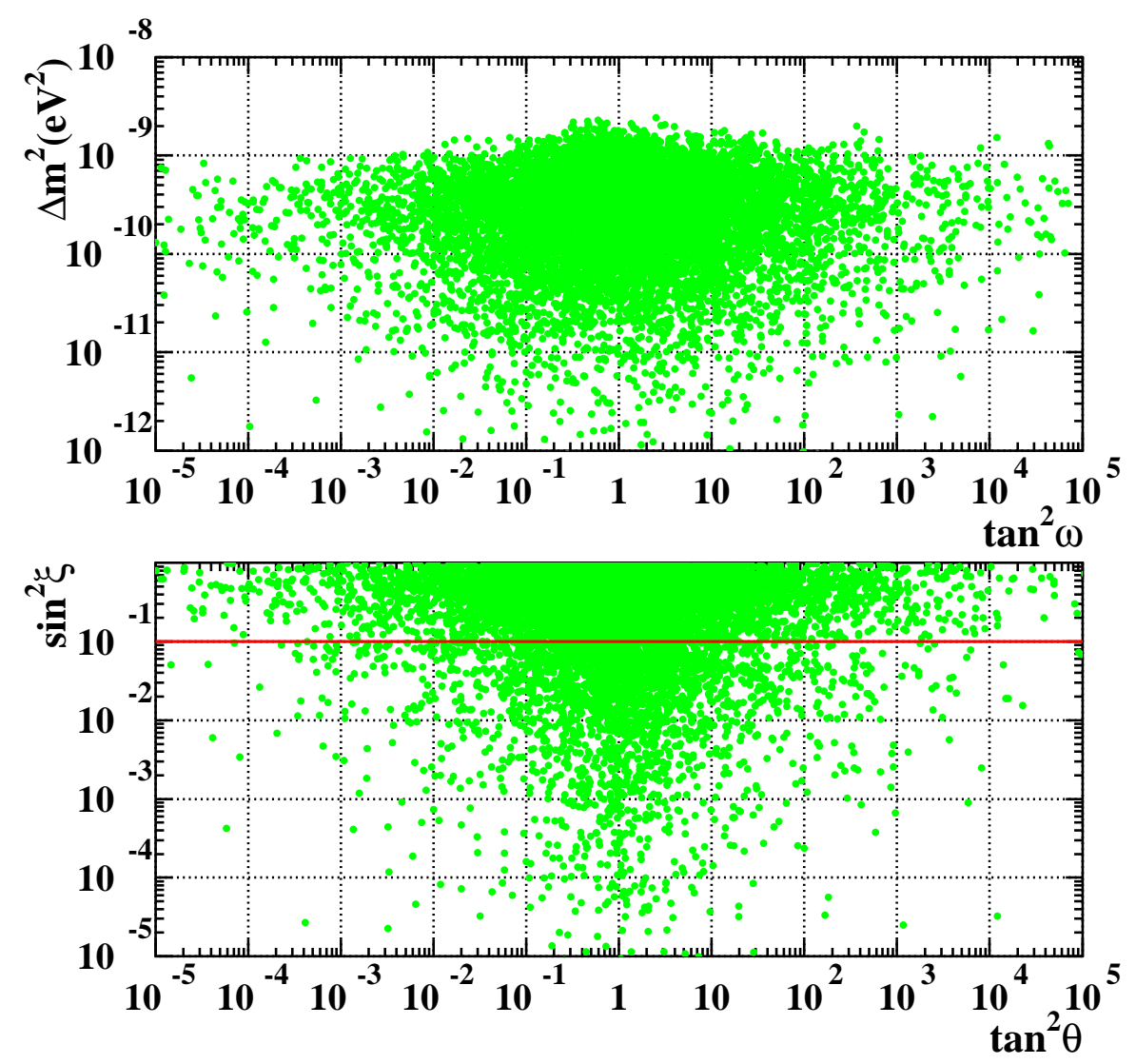

Figure 2: 10000 values of $\Delta m_{\odot}^{2}$ and $\tan ^{2} \omega$ (top), and $\sin ^{2} \xi \tan ^{2} \theta$ (bottom) obtained by randomly generated elements for the $M_{1}$ matrix and $U_{e 3}, U_{\mu 3}$, for $m_{3}=0.05 \mathrm{eV}$ (see text). The solid line indicates $\sin ^{2} \xi=0.1$, which is a good approximation for the upper bound on $\left|U_{e 3}\right|^{2}$ from reactor, solar and atmospheric neutrino data [9].

the atmospheric neutrino data but, to some extent, also the reactor bounds [32]. Fig. 2 depicts 10000 values for the two "solar" neutrino oscillation parameters $\left(\Delta m_{\odot}^{2}\right.$ and $\tan ^{2} \omega$, as before) and the parameters $\sin ^{2} \xi$ and $\tan ^{2} \theta$, where $\theta$ and $\xi$ are the "atmospheric" and "reactor" angles, respectively, when not only $\lambda^{i j}$ but also $U_{e 3}$ and $U_{\mu 3}$ and the sign of $U_{\tau 3}$ are generated at random (the absolute value of $U_{\tau 3}$ is determined by $\sum_{i}\left|U_{i 3}\right|^{2}=1$ ). The only input to the mass matrix is the value of $m_{3}=0.05 \mathrm{eV}$, which is chosen such that the atmospheric data is satisfied. The scatter plots in Fig. 2 can be directly compared with the latest global three-neutrino analysis of the neutrino oscillation data [9]. Note that, even though large values for $\sin ^{2} \xi$ are preferred, a significant fraction of the points falls within the allowed region $\sin ^{2} \xi \lesssim 0.1$.

We summarize this section by restating that, as expected, the minimalistic neutrino 
mass model can easily account for the solar and atmospheric neutrino puzzles. The "light" neutrino masses and the "solar" mixing angle $\omega$ come from $v^{2} / M_{P l}$ and order one coefficients, which can be picked almost at random. The "atmospheric" angle $\theta$ and the "reactor" angle $\xi$ are related to the Yukawa couplings of the right and left-handed neutrinos. We have shown that, if chosen randomly, these can easily account for the data. The only required input to the model is the value of $m_{3}=(y v)^{2} / M_{N}$, which has to be chosen such that the atmospheric neutrino data is satisfied.

\section{Leptogenesis}

One of the exciting feature of models with heavy Majorana fermions is the fact that they potentially contain all the ingredients required to explain the small but nonzero baryonto-entropy ratio of the universe: $n_{B} / s \sim 10^{-10}$. This is accomplished by the mechanism of leptogenesis [33]. In a nutshell [34], because the heavy "right-handed neutrinos" are Majorana particles, their decays violate lepton number. If these decays also violate $\mathrm{CP}$ and happen out of thermal equilibrium, a net lepton number for the universe is generated. Later, through sphaleron effects [35], the net lepton number is partially transformed into a net baryon number, as long as the lepton number is produced before the electroweak phase transition $\left(T \simeq 10^{3} \mathrm{GeV}\right.$ ). If this is the case, the net baryon-to-entropy ratio has been estimated [36] to be

$$
\frac{n_{B}}{s}=\left(\frac{24+4 N_{H}}{66+13 N_{H}}\right) \frac{n_{B-L}}{s},
$$

where $N_{H}$ is the number of Higgs doublet and $n_{B-L}$ is the amount of baryons minus leptons. In the SM, $N_{H}=1$ and $n_{B} / s=(28 / 79) n_{B-L} / s$, or $n_{B} / s=-(28 / 79) n_{L} / s$, assuming that initially only a net lepton number is generated. In this section, we will discuss whether our minimalistic model is capable of generating the observed baryon-toentropy ratio.

In order to estimate "how much" lepton number is produced during right-handed neutrino decays, we compute 34

$$
\epsilon=\frac{\Gamma\left(N \rightarrow l^{3} H\right)-\Gamma\left(N \rightarrow \bar{l}^{3} H^{*}\right)}{\Gamma\left(N \rightarrow l^{3} H\right)+\Gamma\left(N \rightarrow \bar{l}^{3} H^{*}\right)} .
$$

A nonzero value for $\epsilon$ arises from the interference between the tree-level and the absorptive part of the one-loop diagram in Fig. 3, and is given by

$$
\epsilon=\frac{\Im\left(y_{3}^{2} \lambda_{33}^{*}\right)}{\left|y_{3}\right|^{2} 8 \pi} \frac{M_{N}}{M_{P l}} \simeq \frac{m M_{N}}{8 \pi v^{2}} \sin \delta,
$$



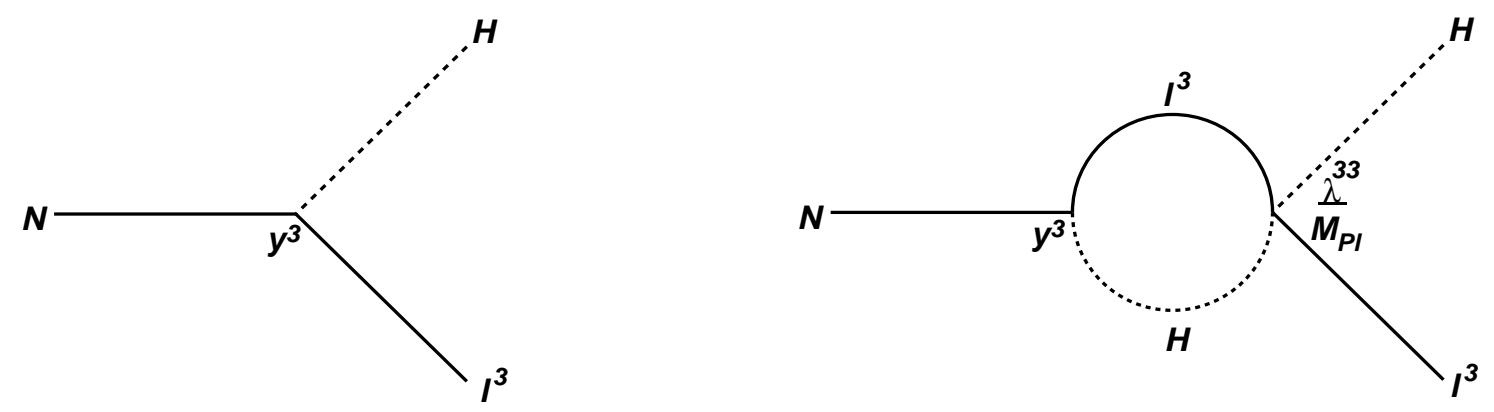

Figure 3: Feynman diagrams for the decay $N \rightarrow l^{3} H:($ A) dominant tree-level contribution and (B) one-loop contribution containing the $M_{P l}$-suppressed operator.

where $y_{3}$ is defined as the Yukawa coupling between the SM singlet-fermion $(N)$, and the appropriate linear combination of SM lepton doublets (i.e. $\left.\mathcal{L} \supset y_{3} L_{3} N H\right), \lambda_{33}$ is the coefficient of the $1 / M_{P l}$ operator when the lepton doublets are expressed in a basis which contains $L_{3}, m=O\left(10^{-5}\right) \mathrm{eV}$ is the light neutrino mass, $v$ is the SM Higgs vev, $M_{N}$ is the Majorana mass of the right-handed state, and $\delta$ is the phase of $y_{3}^{2} \lambda_{33}^{*}$. Numerically

$$
\epsilon=10^{-7}\left(\frac{m}{10^{-5} \mathrm{eV}}\right)\left(\frac{M_{N}}{10^{13} \mathrm{GeV}}\right)\left(\frac{\sin \delta}{1}\right) .
$$

Note that a nonzero value for $\epsilon$ is obtained, even though there is only one right-handed neutrino species. It is clear that, within the renormalizable Lagrangian, it is impossible to generate a net lepton number with just one right-handed neutrino species, due to the absence of $\mathrm{CP}$-violation. However, the presence of the dimension-5 operator solves this problem. This is not at all surprising since the situation here is analogous to having two (or more) right-handed neutrinos with hierarchical Majorana masses (see. e.g., Eq. (3.5)). In this case, it is well known that a net lepton number can be generated via the decay of the lightest right-handed neutrino state.

Is Eq. (4.4) enough to generate the observed baryon-to-entropy ratio? Unfortunately, the answer depends on the history of the very early universe. The simplest hypothesis, perhaps, is to assume that below some very high temperature the universe is described by a thermal bath of all SM particles, including the heavy right-handed neutrino. In this case, the question which must be addressed is whether the right-handed neutrino falls out of thermal equilibrium before decaying. Such a condition is (roughly) satisfied if the 
right-handed neutrino decay width is smaller than the expansion rate of the universe at temperatures $T \sim M_{N}$. From Fig. $3(\mathrm{~A})$, the decay width of the right-handed neutrino is

$$
\Gamma=\frac{y_{3}^{2}}{8 \pi} M_{N} \simeq \frac{m_{3} M_{N}^{2}}{8 \pi v^{2}}
$$

where $m_{3} \simeq\left(y_{3} v\right)^{2} / M_{N}$ is the heaviest neutrino mass, chosen to account for the atmospheric mass scale. In a radiation dominated universe $\left(H \simeq 1.7 g_{*}^{1 / 2} T^{2} / M_{P l}\right)$, the out-of-equilibrium constraint implies

$$
m_{3} \lesssim 10^{-3} \mathrm{eV}
$$

which is about two orders of magnitude below the required $m_{3} \sim 0.05 \mathrm{eV}$, implying that the right-handed neutrinos do not decay sufficiently out of thermal equilibrium and that the leptogenesis mechanism cannot generate the observed baryon-to-entropy ratio. More detailed estimates reach the same conclusion. For example, the authors of Ref. [37] estimate that values of $\epsilon \sim 10^{-5}$ are required for values of $m_{3} \lesssim 10^{-2} \mathrm{eV}$, which cannot be satisfied by our minimalistic model.

One simple way of circumventing this is to assume a different history for the early universe. In particular, if the right-handed neutrinos are never in equilibrium with the thermal bath of light states, the above constraint does not apply. Estimates of the number density of right-handed neutrinos at the time of their decay and the resulting baryon-toentropy ratio are very model dependent, and require that we go beyond the minimalistic model (indeed, we have nothing to say about inflation, for example). Nonetheless, it is worth mentioning that, for example, in a number of inflationary models, right-handed neutrinos may be produced at the time of reheating even if the reheating temperature $T_{R H}$ is (much) lower than its mass (see 37 and references therein for details). In this case, they are almost always (way) out of equilibrium when they decay, and the estimates for the generated baryon-to-entropy ratio can be drastically different. Two possibilities were analyzed in detail in [37]. First, the right-handed neutrinos may be coupled to the inflaton field and therefore are still produced at the time of reheating even if $T_{R H}<M_{N}$, as long as they are lighter than the inflaton field itself. Second, the right-handed neutrinos may be produced during preheating [38], even with relatively low reheating temperatures $\left(M_{N} \gg T_{R H}\right)$ due to the non-perturbative decay of large inflaton oscillations during reheating. This mechanism is particularly efficient for fermions [37. In both cases it seems plausible that our minimalistic neutrino mass model can generate the observed baryon-to- 
entropy ratio. According to estimates of [37], this will happen as long as $M_{N} \gtrsim 10^{13} \mathrm{GeV}$ and the reheating temperature is "large" $\left(T_{R H} \gtrsim 10^{10} \mathrm{GeV}\right)$.

\section{Discussions and Conclusions}

The SuperKamiokande atmospheric neutrino data strongly suggest that effects beyond the standard model of particle physics are present in the leptonic sector. The simplest extension of the SM which explains the atmospheric neutrino data is to assume that neutrinos are massive, and that they mix. In light of this strong experimental evidence, it is natural to assume that the long standing solar neutrino puzzle is also solved by neutrino oscillations.

In this paper, we have emphasized that nonrenormalizable $1 / M_{P l}$ operators provide a solution to solar neutrino puzzle without the addition of any new physics. On the other hand, the atmospheric neutrino puzzle requires the existence of new physics at intermediate energy scales. The most "economical" model requires one new field at this new physics scale, which can be anywhere between $10^{9} \mathrm{GeV}$ and $10^{14} \mathrm{GeV}$ for typical Yukawa coupling values. It is important to note that, as far as solving the neutrino puzzles is concerned, this simple model is just as successful as other more complex models. A potential drawback of the model is that mixing angles are not predicted, so that the required relative magnitudes of the Yukawa coupling has to be put in by hand in order to satisfy the atmospheric neutrino data. In particular the right-handed neutrino needs to couple to $\nu_{\mu}$ and $\nu_{\tau}$ with equal strength, while the coupling to $\nu_{e}$ has to be somewhat suppressed. However, as we have shown (Fig. 2), the present experimental constraints on $\left|U_{e 3}\right| \lesssim 0.3$ can still be accommodated without difficulty by assuming that the Yukawa couplings are picked completely at random, in the spirit of neutrino mass "anarchy" [32]. Within this perspective, all mixing angles easily arise from random Yukawa couplings and random coefficients for the $1 / M_{P l}$ operator, and the only input to the model is the value of $m_{3}$. This situation may well change in the near future if $U_{e 3}$ is constrained to be very small.

The idea that the solar neutrino puzzle may be evidence for "quantum gravity" effects will be severely tested in the near future. The "just-so" solution $\left(\Delta m_{\odot}^{2} \lesssim 10^{-10} \mathrm{eV}^{2}\right)$ is already somewhat disfavoured by the solar neutrino data [9], while values of $\Delta m_{\odot}^{2} \gtrsim$ $5 \times 10^{-10} \mathrm{eV}^{2}$ are still acceptable [9] in the recently revealed "quasivacuum" region [39]. 
However, the Borexino experiment, which is to start data taking next year, will thoroughly explore $\Delta m_{\odot}^{2} \lesssim 5 \times 10^{-9} \mathrm{eV}^{2}$ and large mixing in a standard solar model independent way [40]. It is safe to say that, after a few years, these values of $\Delta m_{\odot}^{2}$ will be either confirmed or definitively ruled out. Furthermore, the minimalistic model also predicts hierarchical neutrino masses, such that the sign of the "atmospheric" mass-squared difference $\Delta m_{\mathrm{atm}}^{2}$ is positive. This possibility will be definitively tested at a future neutrino factory 441.

\section{Acknowledgements}

We would like to thank Martin Hirsch, Steve King, Magda Lola, and Apostolos Pilaftsis for useful and enlightening conversations, while AdG acknowledges the hospitality of IFIC during his stay in València, where this work was initiated. This work was supported by DGICYT under grant PB98-0693 and by the TMR network grants ERBFMRXCT960090 and HPRN-CT-2000-00148 of the European Union.

\section{References}

[1] SuperKamiokande Collaboration, Y. Fukuda et al., Phys. Lett. B433, 9 (1998); Phys. Lett. B436, 33 (1998); Phys. Lett. B467, 185 (1999); Phys. Rev. Lett. 82, 2644 (1999). H. Sobel, talk at XIX International Conference on Neutrino Physics and Astrophysics, Sudbury, Canada, June 2000 (http://nu2000.sno.laurentian.cd); T. Toshito, talk at the XXXth International Conference on High Energy Physics, July 27 - August 2, 2000 (ICHEP 2000) Osaka, Japan (http://www.ichep2000.rl.ac. $u k$.

[2] Y. Suzuki, talk at XIX International Conference on Neutrino Physics and Astrophysics, Sudbury, Canada, June 2000 (http://nu2000.sno.laurentian.cd); T. Takeuchi, talk at the XXXth International Conference on High Energy Physics, July 27 - August 2, 2000 (ICHEP 2000) Osaka, Japan (http://www.ichep2000.rl.ac. uk). Homestake Collaboration, B.T. Cleveland et al., Astrophys. J. 496, 505 (1998); R. Davis, Prog. Part. Nucl. Phys. 32, 13 (1994); K. Lande, talk at XIX International Conference on Neutrino Physics and Astrophysics, Sudbury, Canada, June 2000 (http://nu2000.sno.laurentian.cd); SAGE Collaboration, J.N. Abdurashitov et al., Phys. Rev. C60, 055801 (1999); V. Gavrin, talk at XIX International Conference 
on Neutrino Physics and Astrophysics, Sudbury, Canada, June 2000 (http://nu2000.sno.laurentian.cd). GALLEX Collaboration, W. Hampel et al., Phys. Lett. B447, 127 (1999). E. Belloti, talk at XIX International Conference on Neutrino Physics and Astrophysics, Sudbury, Canada, June 2000 (http://nu2000.sno.laurentian.ca).

[3] R. Becker-Szendy et al., Phys. Rev. D46, 3720 (1992). H.S. Hirata et al., Phys. Lett. B280, 146 (1992); Y. Fukuda et al., ibid B335, 237 (1994). W.W.M. Allison et al., Phys. Lett. B449, 137 (1999). A. Mann, talk at XIX International Conference on Neutrino Physics and Astrophysics, Sudbury, Canada, June 2000 http://nu2000.sno.laurentian.ca/T.Mann/index.htm 2000, http://www.ichep2000.rl.ac.uk/Program.htm, See also hep-ex/0001058, Proc. of the Sixth International Workshop on Topics in Astroparticle and Underground Physics, TAUP99, Paris September 1999.

[4] O. Miranda, et al., hep-ph/0005259 (Nucl. Phys. B in press).

[5] S. Bergmann, et al., Phys. Rev. D62, 073001 (2000).

[6] N. Fornengo, M.C. Gonzalez-Garcia, and J.W.F. Valle, JHEP 0007, 006 (2000); M.C. Gonzalez-Garcia et al., Phys. Rev. Lett. 82, 3202 (1999) and references therein.

[7] For a recent review on the phenomenology of neutrino oscillations see,e.g., S.M. Bilenky, C. Giunti, and W. Grimus, Prog. Part. Nucl. Phys. 43 (1999) 11.

[8] J.W.F. Valle, Phys. Lett. B199 (1987) 432.

[9] M.C. Gonzalez-Garcia, et al., hep-ph/0009350 and references therein.

[10] D.E. Groom et al., Eur. Phys. J. C15, 1 (2000).

[11] L. Baudis et al., Phys. Rev. Lett. 83, 41 (1999).

[12] LSND Collaboration, C. Athanassopoulos et al., Phys. Rev. Lett. 75 (1995) 2650; Phys. Rev. Lett. 77 (1996) 3082; Phys. Rev. Lett. 81 (1998) 1774.

[13] J. T. Peltoniemi, D. Tommasini, and J. W. F. Valle, Phys. Lett. B298 (1993) 383; D. O. Caldwell and R. N. Mohapatra, Phys. Rev. D48 (1993) 329; J. T. Peltoniemi and J. W. F. Valle, Nucl. Phys. B406 (1993) 409 [hep-ph/9302316]. 
[14] A. Ioannisian and J.W.F. Valle, hep-ph/9911349; M. Hirsch and J.W.F. Valle, hepph/0009066, to appear in Phys. Lett. B.

[15] J. Schechter and J. W. F. Valle, Phys. Rev. D22, 2227 (1980).

[16] For reviews on the theory of neutrino masses see, e.g., J.W.F. Valle, "Neutrino Masses: From Fantasy to Facts," Springer Tracts in Modern Physics 163 (2000) 35-68, hep-ph/9906378 and Prog. Part. Nucl. Phys. 26 (1991) 91.

[17] Y. Hayato et al., Phys. Rev. Lett. 83, 1529 (1999).

[18] R. Barbieri, J. Ellis, and M.K. Gaillard, Phys. Lett. B90, 249 (1980).

[19] E.Kh. Akhmedov, Z.G. Berezhiani and G. Senjanovic, Phys. Rev. Lett. 69, 3013 (1992).

[20] M. Gell-Mann, P. Ramond, R. Slansky, in Supergravity, ed. P. van Niewenhuizen and D. Freedman (North Holland, 1979); T. Yanagida, in KEK lectures, ed. O. Sawada and A. Sugamoto (KEK, 1979); R.N. Mohapatra and G. Senjanovic, Phys. Rev. Lett. 44912 (1980).

[21] I. Antoniadis, Phys. Lett. B246 (1990) 377; N. Arkani-Hamed, S. Dimopoulos, and G. Dvali, Phys. Let. B429, 263 (1998); I. Antoniadis, et al., Phys. Lett. B436, 257 (1998) hep-ph/9804398; N. Arkani-Hamed, S. Dimopoulos, and G. Dvali, Phys. Rev. D59,086004 (1999).

[22] M. Hirsch et al., hep-ph/0004115 to appear in Phys. Rev. D; J.C. Romão, et al., Phys. Rev. D61 (2000) 071703 and references therein.

[23] K.S. Babu, J.C. Pati, and F. Wilczek, Nucl. Phys. B566, 33 (2000) and references therein.

[24] N. Arkani-Hamed, et al., hep-ph/0006312.

[25] K.R. Dienes, E. Dudas, and T. Gherghetta, Nucl. Phys. B557, 25 (1999); N. ArkaniHamed, et al., hep-ph/9811448; N. Arkani-Hamed, S. Dimopoulos, hep-ph/9811353.

[26] J. Schechter and J.W.F. Valle, Phys. Rev. D21, 309 (1980).

[27] S. Davidson and S. King, Phys. Lett. B445, 191 (1998). 
[28] S. King, Phys. Lett. B439, 350 (1998).

[29] A. Santamaria and J.W.F. Valle, Phys. Lett. B195, 423 (1987).

[30] K.S. Babu and E. Ma, Phys. Rev. Lett. 61, 674 (1988).

[31] M. Apollonio et al., Phys. Lett. B466 415 (1999) hep-ex/9907037; F. Boehm et al., hep-ex/9912050.

[32] L. Hall, H. Murayama, and N. Weiner, Phys. Rev. Lett. 84, 2572 (2000); N. Haba and H. Murayama, hep-ph/0009174.

[33] M. Fukugita and T. Yanagida, Phys. Lett. B174, 45 (1986).

[34] For recent reviews, see A. Pilaftsis, Int. J. Mod. Phys. A14, 1811 (1999); W. Buchmüller and M. Plümacher, Phys. Rep. 320, 329 (1999); hep-ph/0007176.

[35] V.A. Kuzmin, V.A. Rubakov, and M.E. Shaposhnikov, Phys. Lett. B155, 36 (1985).

[36] S.Y. Khlebnikov and M.E. Shaposhnikov, Nucl. Phys. B308, 885 (1988); J.A. Harvey and M.S. Turner, Phys. Rev. D42, 3344 (1990).

[37] G.F. Giudice et al., JHEP 9908, 014 (1999).

[38] L.A. Kofman, A.D. Linde, and A.A. Starobinsky, Phys. Rev. Lett 73, 3195 (1994).

[39] A. Friedland, Phys. Rev. Lett. 85, 936 (2000); A. de Gouvêa, A. Friedland, and H. Murayama, Phys. Lett. B490, 125 (2000); E. Lisi et al., hep-ph/0005261.

[40] A. de Gouvêa, A. Friedland, and H. Murayama, Phys. Rev. D60, 093011 (1999).

[41] V. Barger, et al., Phys. Lett. B485 (2000) 379 [hep-ph/0004208; A. De Rujula, M.B. Gavela, and P. Hernandez, Nucl. Phys. B547 (1999) 21 hep-ph/9811390; M. Freund, P. Huber, and M. Lindner, Nucl. Phys. B585 (2000) 105 hep$\mathrm{ph} / 0004085$. 